See discussions, stats, and author profiles for this publication at: https://www.researchgate.net/publication/260599142

\title{
Long-term evaluation of a social Robot in Real Homes
}

Conference Paper · April 2014

\section{CITATIONS}

19

3 authors:

Maartje M.A. de Graaf

Utrecht University

36 PUBLICATIONS 554 CITATIONS

SEE PROFILE

Jan A.G.M. Van Dijk

University of Twente

111 PUBLICATIONS 7,769 CITATIONS

SEE PROFILE

Some of the authors of this publication are also working on these related projects:

Human-Robot Relationships and the Good Life View project

Blended Smoking Cessation Treatment View project
READS

559

Somaya Ben Allouch

University of Twente

72 PUBLICATIONS 612 CITATIONS

SEE PROFILE 


\title{
Long-term evaluation of a social robot in real homes
}

\author{
Maartje M.A. de Graaf ${ }^{1}$, Somaya Ben Allouch ${ }^{1}$ and Jan A.G.M. van Dijk ${ }^{1}$
}

\begin{abstract}
As the employment of robots for long-term evaluations in home settings are just starting to be robust enough for research purposes, our study aims at contributing to humanrobot interaction research by adding longitudinal findings to a limited number of long-term social robotics home studies. We placed 70 commercially available robots within people's homes for a period up to six months. In this paper, we report on the collected questionnaire data from 102 people living in these houses. The participants evaluated the robot and their user experiences at six points in time. We observed a mere-exposure effect which causes people to evaluate a novel stimuli more positively when they gain experience and get familiar with it.. The participants evaluated several aspects of the robot. We found user experience initially dropped before rising again when the robot was used over a longer period of time.
\end{abstract}

\section{INTRODUCTION}

Social robotics research is increasingly paying attention to the domestic environment as a context of use. However, this area of research is still in its infancy as only recently robotics technology has become robust enough to allow for the employment of long-term evaluations in home settings. Moreover, an extra challenge for research in these settings lies within privacy issues and a consequent lack of control over users' activities with the robot. Nevertheless, domestic use of robots is already a reality with the arrival of commercial products such as robotic vacuum cleaners and robotic toys.

Although there are many human-robot interaction studies researching people's initial reactions and attitudes towards robots, the temporal dimension of the acceptance process is underexposed. Even today, little is known about the long-term acceptance and use of robots in domestic environments. Yet, people's perceptions on social robot acceptance are likely to change over time when they develop experiences with the robot or their own skills change [34]. Long-term interaction with social robots can be considered as a sub-area of HRI research that investigates the development of interaction patterns between users and social robots over time [21]. It is scientifically acknowledged that long-term effects exist in technology usage and that sustained usages patterns change over time [30]. Despite this recognition, knowledge about these long-term effects in the field of social robotics is still scarce. Up until now, only a few studies investigated the domestic use of robots over a longer period of time [5, 7, 9, 10, 30, 31]. However, studying long-term use and patterns of use is necessary to provide insight into how social robots become part of people's daily routines and to

\footnotetext{
${ }^{1}$ Dept. of Media, Communication \& Organization, Univ. of Twente, Drienerlolaan 5, 7522 AE Enschede, The Netherlands. Email: \{m.m.a.degraaf, s.benallouch, j.a.g.m.vandijk\}@utwente.nl
}

inform designers how to create robots that remain useful beyond the initial adoption [8]. Moreover, studies within people's natural environments can provide practical insights into the continuous use of and user experiences with these robotic products. Longitudinal studies are extremely fertile to explore changes in use behavior and user experience over time.

Even the traditional technology acceptance literature is lacking a profound body of long-term research despite its long history within the information systems literature [32] that continues to receive much attention today. While earlier technology acceptance research mainly focus on explaining why people initially adopt technologies, only a minority of these studies pays attention to what happens in the post-adoption situation. This is where people decide between continuing and discontinuing the use of these technology. Support for differences between pre-adoption and post-adoption beliefs has been provided by the cognitive dissonance theory [6], which claims that the use of a product may change one's perceptions, attitudes, and needs with respect to the use of that product. Consequently, beliefs after using a product may not be the same as the set of beliefs that have led to the initial adoption. Only when people are willing to continue to use a technology after initial adoption, one could assume that the acceptance is a success. It is very likely that these patterns of changing beliefs also happen when using social robots in the home.

The contribution of this paper is to add longitudinal findings to a limited number of long-term studies in human-robot interaction research. The goal is to provide in-depth insight into the changing evaluations of a domestic social robot that is utilized into people's own homes for a longer period of time. By studying social robot acceptance over time, a better understanding of the formation of the users' perceptions towards the robot can be gathered, which, in turn, can help to better shape the design and implementation of social robots.

\section{LONG-TERM ACCEPTANCE}

An alternative to the commonly used adoption models in technology acceptance literature is offered by the domestication theory [29] and the diffusion of innovations theory [28]. Domestication theory is essentially about giving technology a place in everyday life. Here, acceptance is not assumed as a rational, linear, mono-causal and technologically determined process. The domestication theory acknowledges the complexity of everyday life and the place of technology within its dynamics, rituals, rules, routines and patterns. This initially enables researchers to understand technology use in the complex structures of everyday life settings, with attention to interpersonal relationships, social background, changes and continuities. Moreover, it takes into account the increasingly complex interconnection between different technologies, and the 


\begin{tabular}{r|l|l|l|l}
\hline Pre-adoption & Adoption & Adaptation & Incorporation & Identification \\
\hline
\end{tabular}

Figure 1. Timeline of the acceptance phases

convergence of different media technologies and media texts [15]. The diffusion of innovations theory also comprises a process of long-term acceptance. It describes acceptance as the sequence of activities an individual passes from gaining initial knowledge of a technology, to forming an attitude towards that technology, making the initial adoption decision which could lead to the implementation of that technology and, eventually to continuing the use of that technology. While the diffusion of innovations theory is useful as it explains how technologies are appropriated, the value of the domestication theory lies in proving insights into how the user assigns meaning and significance to the technology and how users experience this during the acquisition and usage of the technology. Based predominantly on these two theories of long-term acceptance, together with some other studies on long-term technology use, we formulated five acceptance phases (see figure 1): preadoption, adoption, adaptation, incorporation and identification. In the following, we will describe these acceptance phases in more detail.

Pre-adoption. In the pre-adoption phase, people learn about the technology, determine its value and form expectations and attitudes towards it before they invite the technology into their homes [30, 31]. People want to know more about the technology as they want to learn about the technology's purpose, try to understand the functionality of the technology, and finally pursue to rationalize its internal processes. After this rationalization process comes affection and people will form their interpersonal attitude towards the technology [28]. This is where people become psychologically involved and actively try to gain knowledge about the technology and judge this knowledge accordingly. However, people who are typically unsure are more likely to seek reinforcement in the opinions of others [28]. This may indicate that people who are hesitant to use robots are more sensitive to social influence. Another study exploring the long-term use of mobile phones defines the preadoption as the phase in which people anticipate future experiences by forming expectations [17]. The pre-adoption phase is thus all about the anticipation and preparation of obtaining a technology.

Adoption. The adoption phase contains the decision that leads to the adoption (or rejection) of a technology. This is where people actually start using the technology and gain their first serious user experiences with it [30,31]. This is after some possible initial trials, and most people prefer these trials [28]. However, uncertainly still exists about the expected consequences. People still want to learn about the technology and get familiar with it [17]. The adoption phase is thus about the exploration of the newly obtained technology.

Adaptation. The adaptation phase starts directly after the initial adoption decision has been made. People are still obtaining their initial user experiences, but already have a broad and vague idea of what the technology is all about. People are still pervaded by feelings of excitement as well as frustration, because they experience novel features and encounter learnability flaws [17]. People try to familiarize themselves with the technology, identify any issues or concerns and sometimes show the technology to others [4]. They will explore the technology's complexities and compatibilities in their personal spaces and make necessary changes to enable the technology to be incorporated into their daily routines [28, 30, 31]. As people continue to be curious about and aware of the presence of the technology [4] and try to appropriate to the technology [29], they will finally come to determine reaffirmation of their initial adoption or rejection of further use [29, 30, 31]. In the adaptation phase, people will explore the purpose of the technology in their natural environment and try to adapt the technology to their daily routines.

Incorporation. In the incorporation phase people start to experience a functional dependency on the technology [17], creating routines of use [29, 30, 31] and fully integrating the technology in their daily lives [4]. During the process of incorporation the technology is completely changed or modified by the user [28] and the technology becomes meaningful in people's daily lives [17]. They no longer notice the presence of the technology in their homes as long as it no longer has their primary attention [4]. When the technology allows its users to personalize it, this shapes the possibilities for long-term use, which increases acceptance [1]. However, it could be that the technology is used differently from the way it was intended by designers [29]. In the incorporation phase people have entirely incorporated the technology into daily routines.

Identification. In the identification phase, the technology exceeds its functional purpose and becomes a personal object as people get emotionally attached to it. They accept the technology in their daily lives and it participates in their social interactions. The technology might even communicate parts of their selfidentity that serve to either differentiate them from others or connects them to other by creating a sense of community [17]. In this phase, the technology can become a tool for making status claims or for expressing a specific lifestyle to family, friends and neighbors [29]. The personal side of identification, e.g. personalizing the technology and creating daily routines of use, increases over time. The social side of identification, e.g. enabling self-expression and creating a sense of community, initially decreases but also shows a gradual and sustained increase [17]. People, again, seek reinforcement for the initial adoption, and may even reverse this decision if exposed to conflicting messages about the technology [28]. Thus, even in this phase people can decide to discontinue the use of the technology. However, as people try to avoid or at least reduce a stage of dissonance, they will either change their conflicting knowledge, attitudes or behaviors. Dissonance is the uncomfortable feeling an individual experiences when incongruence occurs between attitude and behavior [6]. This uncomfortable feeling can be resolved by either altering personal beliefs (e.g. attitude towards the technology) or performed behavior (e.g. use of the technology). This means that, when users obtain information that argues to discontinue the use of the robot, they will either advocate reasons why they would continue to use the robot or they will stop using the robot. During this phase, users want supportive information that prevents the occurrence of dissonance. The identification phase is about the final decisions about the sustained use of a technology. 


\section{RELATED WORK}

A few studies have been performed on long-term use of robots in home environments. Most of these studies employ commercially available robots. For example, Sung et al. [30, 31] and Fink et al. [7] evaluated how people used and accepted the Roomba vacuum cleaner in their homes, with thirty and eleven participating households respectively. During their visits at five times during a 6-month ethnographic study, several methods besides questionnaire and interview data were used, such as drawings, probing techniques and checklists of activities with the Roomba. Sung et al. [30] argue that two month will be enough to study stable technology use after the novelty effect has faded away. And both references [7, 30, 31] argue that a combination of multiple types of data is preferred to capture people's routines of use and acceptance of the robot. A framework of long-term domestic use was drawn by Sung et al. [31] and confirmed by Fink et al. [7] containing the key interaction patterns when households accept robots: pre-adoption, adoption, adaptation, and use and retention (similar to the incorporation phase in section 2). On several evaluation measures, participants rated the vacuum cleaner robot more negatively after the introduction [7], but those people who still used the robot after six months were more positive than those who stopped earlier.

Fernaeus et al. [5] reported on a study evaluating the Pleo dinosaur robot with six families with children for two to six months (each family was allowed to stop using the robot at their own terms). The researchers used interviews and video-material recorded by the families, and focused on whether the evaluation of the robot would be affected by prior expectations. Results show that high prior expectations were not met, which caused some participants to stop using the robot when the novelty-effect wear off. Even though the participant initially regarded the robot as a real pet (e.g. petting it, giving it a name and displaying emotions towards it), the disappointing interaction capability of the robot resulted in it being treating as a regular toy. The researchers provided a set of design challenges which are relevant for robotic toys but also for long-term technology use in general.

Another study employed the bunny-shaped Nabaztag robot, the predecessor of Karotz, and video-taped the interaction between elderly users and the robot in three consecutive iterations of each 10 days [26]. The robot initiated several dialogs per day with the goal to stimulate physical activity. The researchers analyzed the verbal and nonverbal behavior of the users. Results show that people only show facial expression under certain circumstances, such as the system working properly without any delays or interruptions. However, they observed many differences in behavior between the six participants. Two participants treated the robot as a companion, gave it a name and missed it when it was gone. But other two participants treated the robot as a tool, had very precise prior expectations which resulted from their backgrounds and experiences with health-related systems. The other two participants had varying opinions of the robot. Overall, the researchers conclude that users are willing to interact and even converse with a robot despite its perceptive and expressive limitations.

The bunny-shaped Karotz robot was used to compare weather information provided by this robot and a tablet display [24]. In total, 32 participants used and evaluated the tablet in week one, the robot in week two, the tablet again in week three, and the robot again in week four. Results show that people liked and used the robot more than the tablet, but their capabilities of using both systems increased over time. Moreover, although not significantly different, the participants had higher preferences for the personalized information provided by the robot rather than the tablet.

The existing studies described above each provide interesting findings on long-term human-robot interaction. However, two of these studies [24, 26] did not last long enough to measure sustained use after the novelty effect as indicated by Sung et al. [30]. Fernaeus et al. [5] employed the Pleo robot, which is rather toy-like and does not have a utilitarian purpose. Other studies did not focus on individual user acceptance, but took a more sociological perspective by looking into the general opinion of the household [5, 7, 30,31]. Moreover, none of these studies included enough participants to allow for the identification of meaningful statistically significant trends in the data.

\section{METHOD}

The aim of the study was to further investigate in a qualitative and explorative way how people's experiences and perceptions of a domestic social robot evolved over time and to study their impact on domestic technology use. In addressing this goal, we employed a commercially available domestic robot with social features. Studying an existing commercial robot has been successfully applied to investigate people's desires and expectations of human-robot interaction and was argued to be favored over robotic research prototypes which are often not suitable for long-term studies in naturalistic environments [5]. Therefore, the choice for an existing commercially available domestic robot seemed appropriate for our research goal.

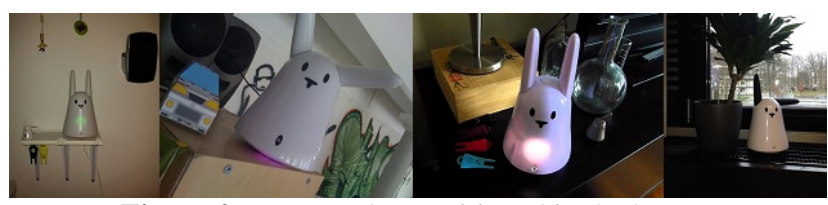

Figure 2. Karotz robot positioned in the homes

Robot. The robot used in this study is Karotz (see figure 2), which is a $30-\mathrm{cm}$ high internet-enabled activated smart bunnyshaped ambient electronic device. Communication occurs via verbal communication, the LED-light in its belly, the moveable ears, and by detecting the presence of other objects nearby. As it is permanently connected to the internet, it is able to react on, transmit, broadcast all types of content available on his network, for example news, messages, music, texts, alerts, and radio. The build-in webcam enables users to communicate with family member at home or to keep an eye on their homes when away. More information on the robot can be found at http://store.karotz.com/en_WW/. We employed 70 Karotz robots into people's homes. Each robot was installed with a basic set of applications, such as daily news broadcasts, daily local weather reports, favorite radio stations, personalized reminders, and randomly spoken phrases to make the robot being perceived as more autonomous and animate. This basic set of applications ensured us that the user experience was somewhat similar among the participants, or at least initially as some participants chose to adjust these applications to their own needs. Besides the required 
applications, participants were free to install additional applications as they thought would be useful or fun for their households. Almost all participants positioned the robot somewhere in their living room. According to the classification of social robots by Breazeal [2], Karotz would be a social interface robot that provides a 'natural' interface by employing humanlike social cues and communicate modalities, whereas the social behaviour only occurs at the interface level resulting in shallow models of social cognition.

Participants. We recruited participants using various methods, such as word of mouth, advertising in public locations (e.g. libraries, leisure centers and supermarkets), and snowball sampling by asking assigned participants for referrals to other people who might participate. During recruitment, we tried to balance out the households' demographic profiles to seek diversity. Therefore we divided participants into four different types of homes: singles, couples, young families (children aged younger than 12) and mature families (children aged older than 12). The goal was to equalize the participants within each household type. In the end, 28 participants were single, 26 participants lived with a spouse, 23 participants were part of a young family, 15 participants were part of a mature family, and for 10 participants did not provide their household type. Furthermore, to increase homogeneity, most participants lived in the region of our university. In total, 70 robots were employed in people's own homes, which resulted in 160 participants. Of these participants, 102 were able or willing to complete the questionnaires. There were 48 males and 54 females, and their age ranged from 8 to $77(M=37.74, S D=16.87)$. Not all participants finished the complete study, as some stopped using the robot before the end of the project. We divided these participants in three groups: rejecters who stopped using the robot within the adaptation phase (26 participants); adopters who stopped using the robot in the incorporation phase (21 participants); and users who were still using the robot in the identification phase and intended to continue to use the robot even after the study (55 participants).

Instruments. We applied both qualitative and quantitative methods to collect data. Quantitative data was collected through the questionnaires evaluating the robot and the participants' user experiences with it and continued doing this as long as they were still using the robot. The questionnaire consisted of several factors of social robots acceptance divided in attitudinal beliefs, social normative beliefs and control beliefs as described in our previous publication on social robots acceptance which introduced seventeen of these factors [11]. Since we aimed at multiple evaluations, we used the five highest loading items per scale based on our previous data. Moreover, in this study, we added the scales of media influence, trust and cost. As most people do not have direct experiences with real robots and are likely to first think of robots as movie creatures, we adapted the social influence scale [16] to measure the influence of media on people's perceptions on robots. Trust is an important factor influencing the acceptance and use of social robots [13], because autonomous systems take over control of some of the technological processes by actively selecting data, transforming information and making their own decisions [27]. We used McCroskey \& Teven [23] trustworthiness scale to measure the participants trust in the robot. Last, we included cost as it is found to be an influential factor in the technology adoption for private use [3]. The scale from Brown and Venkatesh [3] was adapted to measure the participants' perception of cost. In addition to these twenty explanatory variables, we also measured the outcome variables of use attitude from Heerink et al. [14], use intention from Moon \& Kim [25], actual use with the selfreported usage behavior measure from Venkatesh \& Davis [34] and habit from LaRose \& Eastin [19]. In addition to quantitative data, qualitative data was collected from 21 participants living in different households by means of interviews as long as they were using the robot. In total, 97 interviews, each lasting 30-45 minutes were collected, audio-recorded with the participants permission, and transcribed verbatim. For each interview, the participants were interviewed alone, regardless of whether the person lived alone or not. This approach was chosen, because the fundamentals of the theories used in this study lie within psychology rather than sociology, which means that the focus is on the individual and their experiences with the technology within their household.

Procedure. Our study ran from October 2012 to April 2013 and consisted of six phases in which data was collected. Figure 2 displays the acceptance phases on a timeline. At the beginning of each acceptance phase, we collected both interview and questionnaire data. In the pre-adoption phase, the data was used to explore the participants' prior expectations. The first interview was used to get to know the participants and their households, their daily routines and domestic technology use. At the introduction, after installation and a first interaction with robot for about 15 minutes, the data was used to catch the participants' initial impressions their initial reactions. The data obtained in the remaining acceptance phases was used to investigate how the participants evaluated the robot over time, how it was deployed and how their use patterns changed. From now on, each questionnaire started with the question if the robot was stilled used and why (not).

\section{RESULTS}

In this paper, we will only report on the quantitative data collected through the questionnaires in this study. First, we would like to report on the change in use frequency and duration over time (see table 1). Whereas the frequency of use varies widely in the beginning, in the end most participants never use the robot or at best less than once a week. Most participants use the robot brief moments, mostly even shorter than 5 minutes per occurrence. However, a somewhat stable group of heavy users remains over time.

\begin{tabular}{lrrrr}
\hline Usage & T3 & T4 & \multicolumn{1}{c}{ T5 } & \multicolumn{1}{c}{ T6 } \\
\hline Frequency & & & & \\
Never & 19.2 & 27.3 & 45.5 & 31.8 \\
Less than once per week & 11.5 & 15.9 & 15.2 & 22.7 \\
Once per week & 11.5 & 13.6 & 15.2 & 9.1 \\
A few time per week & 26.9 & 15.9 & 19.6 & 13.6 \\
Once per daily & 9.6 & 11.4 & 2.2 & 9.1 \\
Multiple time a day & 21.2 & 15.9 & 4.3 & 13.6 \\
\hline Duration at a time & & & & \\
Less than 5 minutes & 50.0 & 59.1 & 65.2 & 59.1 \\
5 to 15 minutes & 26.9 & 25.0 & 15.2 & 31.8 \\
15 to 30 minutes & 7.7 & 9.1 & 10.9 & 0.0 \\
30 to 60 minutes & 5.8 & 0.0 & 2.2 & 0.0 \\
More than 60 minutes & 9.6 & 6.8 & 6.5 & 9.1 \\
\hline
\end{tabular}


Table 1. Usage over time (in \%)

\begin{tabular}{|c|c|c|c|c|c|c|c|c|}
\hline Variable & $\begin{array}{c}\text { T1 } \\
M(S D) \\
\end{array}$ & $\begin{array}{c}\text { T2 } \\
M(S D) \\
\end{array}$ & $\begin{array}{c}\text { T3 } \\
M(S D)\end{array}$ & $\begin{array}{c}\mathbf{T 4} \\
M(S D)\end{array}$ & $\begin{array}{c}\text { T5 } \\
M(S D)\end{array}$ & $\begin{array}{c}\mathbf{T 6} \\
M(S D) \\
\end{array}$ & $\begin{array}{c}F \\
(5,96)\end{array}$ & $P$ \\
\hline \multicolumn{9}{|l|}{ Attitudinal Beliefs } \\
\hline Usefulness & $4.35(1.2)$ & $3.64(1.1)$ & $3.06(1.4)$ & $2.64(1.2)$ & $2.89(1.4)$ & $3.55(1.0)$ & 36.007 & .000 \\
\hline Ease of use & $08(1.0)$ & $4.73(0.8)$ & $4.58(1.0)$ & $4.59(1.1)$ & $4.77(1$ & $4.61(1.1)$ & 4.522 & .001 \\
\hline Adaptability & $33(1.2)$ & $3.72(0.8)$ & $3.19(1.0)$ & 2.85 & $3.00(1$ & $4.03(1.3)$ & 35.572 & .000 \\
\hline Intelligence & $24(1.2)$ & $3.93(0$ & $3.53(1.0)$ & 3.59( & 3.82( & $3.63(1.2)$ & & .000 \\
\hline Enjoyment & $89(1.0)$ & $3.93(1$ & $3.82(1$ & 3.21 & & & 36.564 & .000 \\
\hline Attra & & 4.84( & 4.41 & 4.22 & & 3.74 & 33.731 & .000 \\
\hline orphism & & 2.56 & 2.4 & & & 4.02 & 155.756 & .000 \\
\hline Real & & 3.14 & & & & & & .000 \\
\hline & & & & & & & & .000 \\
\hline Companionship & & & & & & & 56.709 & .000 \\
\hline \multicolumn{9}{|l|}{ Social normative beliefs } \\
\hline Social influence & $4.97(1.1)$ & $5.00(0.7)$ & $4.41(1.1)$ & $4.32(1.1)$ & $4.11(1.2)$ & $4.36(1.4)$ & 13.115 & .000 \\
\hline uence & & 3.93( & 4.1 & 3.99 & & & 1.186 & .316 \\
\hline & 1) & 2.26( & 1.65 & 1.62 & 1.90 & 3.07 & 46.089 & .000 \\
\hline Tru & 2) & 4.59( & 4.22( & 4.26( & 4.46 & 3.73 & 64.266 & .000 \\
\hline Attitude towards robots & $3.51(1.1)$ & $3.97(0.9)$ & $3.90(0.9)$ & $3.91(\mathrm{C}$ & 3.88( & 3.72( & 4.052 & .002 \\
\hline \multicolumn{9}{|l|}{ Control beliefs } \\
\hline Behavioral control & $4.40(1.2)$ & $3.65(0.9)$ & 3.91 (1.3) & 3.64 (1.3) & & & 10.582 & .000 \\
\hline Anxiety towards robots & 3.29 (1.3) & $3.70(0.7)$ & $3.23(1$. & $3.51(0.7)$ & 3.83 & 3.65( & 6.542 & .000 \\
\hline Cost & & $5.66(0$ & 5.23( & 5.45( & 5.41 & 4.39 & 27.089 & .000 \\
\hline \multicolumn{9}{|l|}{ Outcome variables } \\
\hline Use attitude & & & & & & & 37.936 & .000 \\
\hline Use intention & $4.31(1.0)$ & $3.60(0.9)$ & $2.97(1.2)$ & $2.64(1.2)$ & $2.96(1.3)$ & $4.05(1.2)$ & 45.386 & .000 \\
\hline Actual use & & & $3.71(1.2)$ & $2.60(1.0)$ & $2.48(1.0)$ & $2.54(0.8)$ & 50.983 & .000 \\
\hline Habit & & & $2.63(1.1)$ & $2.37(1.0)$ & $2.38(1.1)$ & $3.50(1.2)$ & 26.902 & .000 \\
\hline
\end{tabular}

$\mathrm{T} 1=$ pre-adoption, $\mathrm{T} 2=$ introduction, $\mathrm{T} 3=$ adoption, $\mathrm{T} 4=$ adaptation, $\mathrm{T} 5=$ incorporation, $\mathrm{T} 6=$ identification

Table 2. Long-term evaluation of acceptance variables in the different acceptance phases

We used repeated measures ANOVA with time (6 acceptance phases) as a within subjects factor and gender (male vs. female), household type (single vs. couple vs. young family vs. mature family), and user group (rejecters vs. adopters vs. users) as the between subjects factor. Repeated measures ANOVA with more than three time points carries some concerns about the sphericity assumption, which is the assumption of equal variances across groups in between subjects ANOVA. In our data, for most analyses, Mauchly's chi-square test for violations of the sphericity assumption was significant, which indicates non-equal variances. To increase statistical power, the $F$-scores from the Greenhouse-Geisser tests are reported here for all repeated measures ANOVA tests. Moreover, some participants dropped out before the end of the study and therefore did not complete all the questionnaires. Missing values due to this drop-out were replaced with the mean score of that acceptance phase for this analysis, otherwise we would only provide insight to those evaluations obtained from participants who continued to use the robot until the very end of the study. Table 2 presents the results of these analyses. The evaluation of all acceptance variables, besides media influence, significantly changed over time. Overall, prior to the introduction of the robot, most of these evaluations were the most positive ones within the whole project. This means that the participants had higher expectations of the robot which were not met after the participants made their first impressions of the robot. This was especially the case for rejecters. Rejecters had even higher expectations than any other user group. A Bonferroni post-hoc test showed significant main effects for user group on usefulness $(F(1,2)=6.675, p=.002)$, adaptability $(F(1,2)=4.885, p=.009)$, enjoyment $(F(1,2)=4.615$, $p=.012)$, companionship $(F(1,2)=4.119, p=.019)$, social influence $(F(1,2)=4.123, p=.019)$, use attitude $(F(1,2)=5.517$, $p=.005)$, use intention $(F(1,2)=8.607, p<.001)$, actual use $(F(1,2)=10.652, p<.001)$, and habit $(F(1,2)=13.282, p<.001)$. Compared to rejecters and adopters, users evaluated the robot more positively, experiences less social influence, had higher intentions to use the robot, actually used the robot more frequently and had a stronger habitual use of the robot. Additionally, another Bonferroni post-hoc test showed significant main effects of household type on usefulness $(F(1,4)=2.939, p=.024)$, intelligence $(F(1,3)=2.727, p=.034)$, enjoyment $(F(1,3)=3.855, p=.006)$, attractiveness $(F(1,3)=$ $5.634, p>.001)$, realism $(F(1,3)=2.794, p=.030)$, sociability $(F(1,3)=2.524, p=.046)$, and trust $(F(1,3)=5.037, p=.001)$. Compared to singles, couples and young families, participants living in a mature family evaluated several aspects of the robot and their interaction experiences with it more positively.

In addition to the long-term evaluations of the acceptance variables, we also investigated the explanatory power of these acceptance variables within each acceptance phase on the outcome variables of use attitude, use intention, actual use and habit using stepwise multiple regression analysis. Within the preadoption phase, usefulness, enjoyment, social influence and negative attitude towards robots explained for $61 \%$ the 
participants' use attitudes $(F(4,96)=39.896, p<.001)$. And usefulness, enjoyment, anthropomorphism, realism and use attitude explained for $69 \%$ the participants' use intention $(F(5,95)=46.354, p<.001)$. Figure 3 visualizes these results.

At the introduction, both usefulness and social influence explained for $73 \%$ the participants' use attitudes $(F(2,100)=$ $135.252, p<.001$ ). And together usefulness, adaptability, social influence and use attitude explained for $77 \%$ the participants' use intention $(F(4,96)=79.273, p<.001)$. Figure 4 visualizes these results.

Within the adoption phase, together usefulness, adaptability, media influence and perceived behavioural control explained $75 \%$ of the variance of use attitude $(F(4,96)=75.333, p<.001)$. Use intention was for $83 \%$ explained by usefulness, social influence and use attitude $(F(4,96)=162.450, p<.001)$. Actual use was for $73 \%$ explained by ease of use, media influence, trust, perceived behavioral control and habit $(F(5,93)=54.157, p<$ .001). And, together, enjoyment, media influence, image, trust, perceived behavioral control, personal innovativeness and actual use explained $75 \%$ of the variance of habitual use $(F(7,91)=$ $41.864, p<.001)$. Figure 5 visualizes these results.

Within the adaptation phase, together usefulness, ease of use, adaptability, enjoyment, sociability and image explained $81 \%$ of the variance of use attitude $(F(6,90)=70.145, p<.001)$. Use intention was for $85 \%$ explained by usefulness, adaptability, enjoyment, realism, companionship and use attitude $(F(6,90)=$ 90.249, $p<.001)$. Actual use was for 37\% explained by usefulness, social influence, robot related experiences, use attitude and habitual use $(F(5,93)=40.138, p<.001)$. And, together, enjoyment, trust and actual use explained $64 \%$ of the variance of habitual use $(F(3,95)=41.864, p<.001)$. Figure 6 visualizes these results.

Within the incorporation phase, together usefulness, social influence, image and anxiety towards robots explained $87 \%$ of the variance of use attitude $(F(4,92)=112.071, p<.001)$. Use intention was for $90 \%$ explained by usefulness, media influence, image, robot related experiences and use attitude $(F(5,91)=$ 133.690, $p<.001$ ). Actual use was for $79 \%$ explained by usefulness, anthropomorphism, perceived behavioral control, personal innovativeness and habit $(F(5,93)=58.104, p<.001)$. And, together, usefulness, social influence and anxiety towards robots explained $46 \%$ of the variance of habitual use $(F(3,95)=$ $22.059, p<.001)$. Figure 7 visualizes these results.

Within the identification phase, usefulness and media influence explained $33 \%$ of the variance of use attitude $(F(2,96)=14.023, p<.001)$. Use intention was for $59 \%$ explained by adaptability and use attitude $(F(2,96)=39.587, p<$ $.001)$. Actual use was for $63 \%$ explained by usefulness, trust and use intention $(F(3,95)=31.546, p<.001)$. And, both usefulness and cost explained $44 \%$ of the variance of habitual use $(F(2,96)=$ 21.889, $p<.001$ ). Figure 8 on the next page visualized these results.

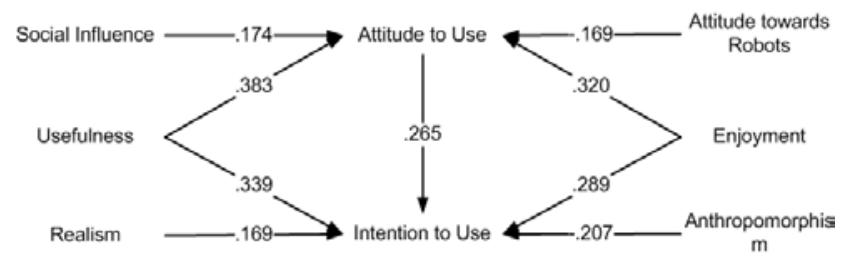

Figure 3. Influential variables within the pre-adoption phase

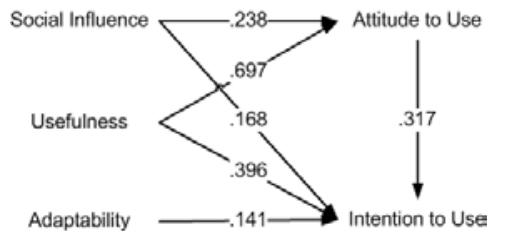

Figure 4. Influential variables at the introduction

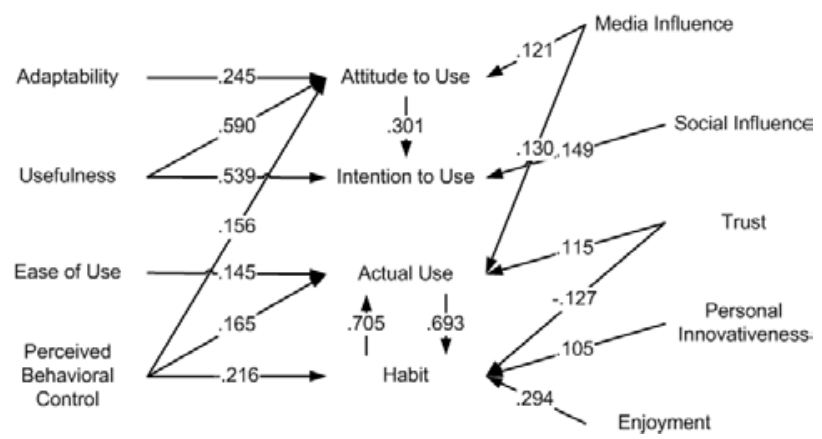

Figure 5. Influential variables within the adoption phase

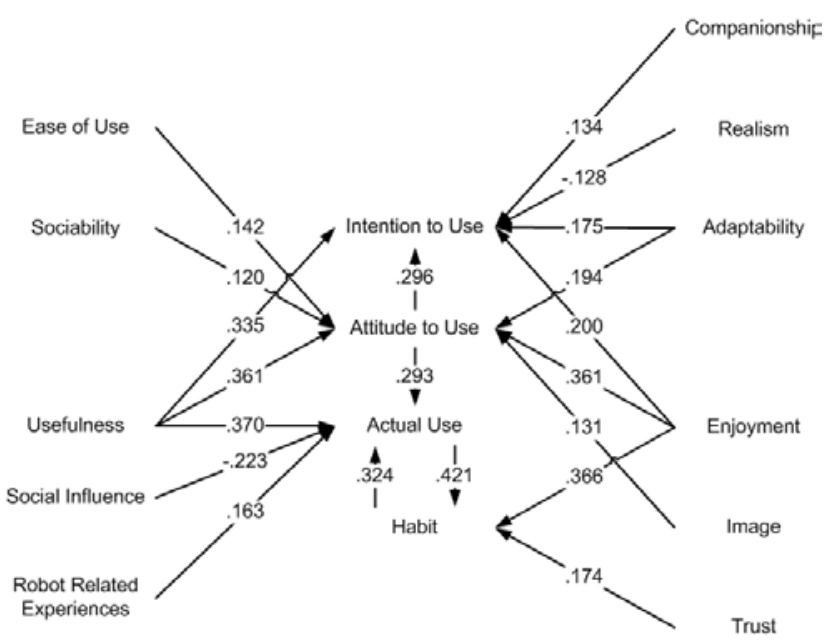

Figure 6. Influential variables within the adaptation phase

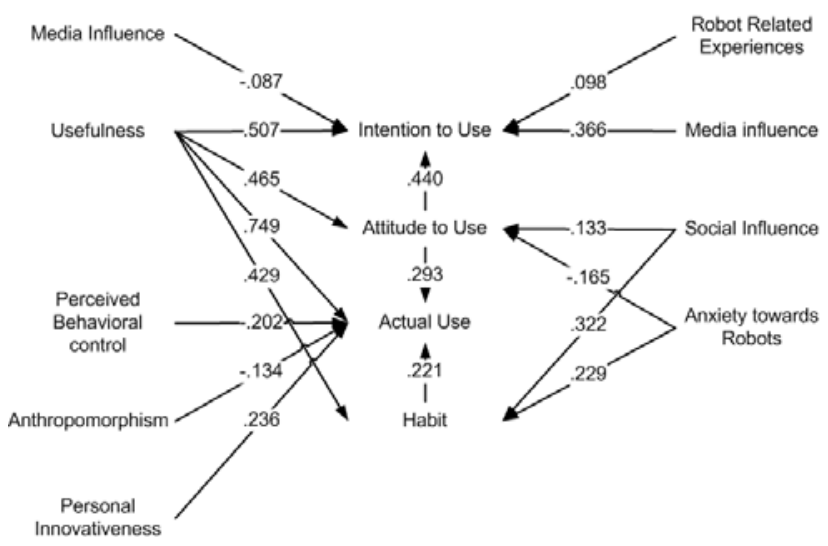

Figure 7. Influential variables within the incorporation phase 


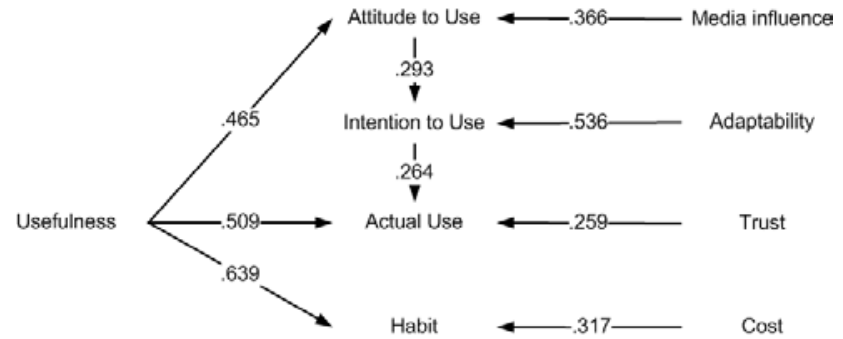

Figure 8. Influential variables within the identification phase

\section{DISCUSSION}

This paper presents a long-term study of social robot acceptance to better understand the users' evaluations of the robot over time, which can help to better shape the design and implementation of social robots.

In the theoretical section, we presented a long-term perspective of long-term technology use in home environments consisting of six acceptance phases. This perspective was based on two prominent theories $[28,29]$ complemented with research results from robots and other types of interaction technologies. A first glance at our interview results seems to confirm these six acceptance phases, however, this data has not been officially analyzed yet. Nevertheless, as these theoretical acceptance phases were based along the results from both robotics and general interaction technology, we believe that these acceptance phases could be applied to research studying long-term use of robots as well as interaction technology in general.

We observed a mere-exposure effect within our data, which is the tendency for novel stimuli to be liked more or rated more positively after someone has been repeatedly exposed to them. For example, earlier findings [33] suggests that system experience influences individual user's playfulness with the system, and that this increase significantly influences an individual's perception of the complexity of the system. Thus, when users first interact with a computer system, they typically feel intimidated and stressed, as a result their degree of playfulness is low. As they obtain some level of familiarity with the system, they are more apt to explore the system and interact spontaneously with it. This is similar to the finding that playfulness mediates the effect of system experience on perceived ease of use [12]. The effect that when people gain experience with a system, they will feel more joy when using that robot and find it more easy to use was also reported in a human-robot interaction study [18] and has been found within our study as well. This result can be explained by a novelty effect in the beginning which fades away after some time, but enjoyment increases again when people see the robot as a familiar interactant. Moreover, familiarizing oneself with a robot causes people to experience more meaningful social interactions with that robot, as earlier findings suggest [18]. A similar pattern is observed within our data. Although initially the evaluation of sociability decreases, at the end this evaluation raises again beyond its initial measures administered before the introduction to the robot.

Rather surprisingly, our findings suggest that people living in mature families evaluated the robot more positively compared to people living alone, with a spouse or with young children. This is in contrast with an earlier short-term study in which participants assigned to the individual interaction condition, compared to participants in the group conditions, developed a stronger sense of responsibility and attachment to the robot might not develop because they did not have to share the robot with other group members [20]. Indeed we had expected that people living alone or people with young children would evaluate the robot more positively. An explanation for our findings could be that teenagers are more willing to experiment with new technologies which resulted in both them and their parents to evaluate the robot more positively.

When looking at all regression analyses, it seems that usefulness is an important acceptance variable for social robot, as it was part of most of the regressions and often the strongest predictor as well. Moreover, the most occurring reason for discontinuance was that the participants could not find an added value for the robot. For example, their smart phone provides similar functionalities and is much easier to carry around. The importance of usefulness was also stressed in an earlier longterm study with the Roomba vacuum cleaner robot [7] and the majority of the households in their study did not perceive the robot as useful.

Limitations. For this study, employing 70 robots in people's own homes over a longer period of time, we had to depend on commercially available robots because research robots are still not robust enough to be studied outside the lab for extended periods of time without supervision of an expert. As commercially available robots still have limited capacities, this might cause a gap between initial expectations and actual experiences after initial interactions with the robot [21]. Our participants also encountered this gap, which has caused some of them to quit using the robot before the end of the study. Similar results were found by Fernaeaus et al. [5], who also reported that, after initial novelty effect, people stopped using the robot due to having high expectation which the robot did not fulfill. Robotic designers should try to bridge this expectation gap by identifying the most important improvements that should be made [22]. The strength of the influence of the acceptance variables in our regression analysis might provide insight into these important aspects. Moreover, this study only reports on regression analyses between the acceptance variables and the outcome variables separately. However, to evaluate the complex indirect effects and interaction effects between the independent variables simultaneously, these should ideally be tested in a complete model using structural equation modeling. This analytical procedure, however, needs a large sample size, which is often a problem in human-robot interaction research. Currently, we are collecting such a large sample of questionnaire data on people's anticipated acceptance of a social robot within their own homes based on the same variables as reported here. The last limitation is that, for this study, we used a zoomorphic robot. Different results may result from studies conducted using other types of robots. Future research should thus try to replicate our results using other types of robots.

\section{CONCLUSIONS \& FUTURE WORK}

This study adds longitudinal findings to a limited number of long-term studies in human-robot interaction research and provides in-depth insight into the process of long-term acceptance of social robots. The main conclusion of our study is that, for most acceptance variables, people's evaluation of a 
robot, after initially dropping, will increase again as they gain experience and get familiar with it.

As this paper only reports on the quantitative data collected during our study, our future work will focus on combing these results with the qualitative data observed during the interviews. Such an approach allows triangulation, which could facilitate the validation of our findings and might help explain why certain effects between the acceptance variables occurred as they did within our data. Moreover, the qualitative data could provide more insight into how the robot was actually used by the participants, e.g. what functionalities were used and with what intentions. This information could, for example, be used to derive design guidelines for future domestic (social) robots. Moreover, the quantitative data allows us to further build our suggested perspective on long-term technology use and the described acceptance phases.

\section{REFERENCES}

[1] T.E. Backer. Finding the balance: Program fidelity and adaptation in substance use prevention. Centre for Substance Abuse Prevention, Washington D.C., USA. (2000).

[2] C.L. Breazeal. Towards sociable robots. Robotics \& Automonous Systems, 42: 167-175. (2003).

[3] S.A. Brown, V. Venkatesh. Model of adoption of technology in households: A baseline model test and extension incorporating household life cycle. MIS Quarterly, 29: 399-426. (2005).

[4] G. Demiris, Parker Oliver, D., Dickey, G., Skubic, M., Rantz, M. Findings from an participatory evaluation of a smart home application for older adults. Technology and Health Care, 16: 111118. (2008).

[5] Y. Ferneaus, M. Hakansson, M. Jacobsson, S. Ljungblad. How do you play with a robotic toy animal?: A long-term study of Pleo. Proc. of the 9th Int. Conf. on Interaction Design and Children, Barcelona, Spain. (2010).

[6] L. Festinger. A theory of cognitive dissonance. Stanford University Press, Stanford, CA, USA. (1957).

[7] J. Fink, V. Bauwens, F. Kaplan, P. Dillenbourg. Living with an vacuum cleaning robot: A 6-months ethnographc study. Int. J. of Social Robotics, 5: 389-408. (2013).

[8] J. Forlizzi, C. DiSalvo. Service robots in the domestic environment: A study of the Roomba vacuum in the home. Proc. of the Int. Conf. on Human-Robot Interaction, Salt Lake City, UT, USA. (2006).

[9] M.M.A. de Graaf, T, Klamer, S. Ben Allouch. Return of Harvey: Acceptance and Use of Social Robots (Work-In-Progress paper). Int. Conf. of Social Robotics, Amsterdam, The Netherlands. (2011).

[10] M.M.A. de Graaf, S. Ben Allouch. Harvey’s last appearance: Longterm use and acceptance of social robots. Int. Communication Association Conf., Phoenix, AZ, USA. (2012).

[11] M.M.A. de Graaf, S. Ben Allouch. Exploring influencing variables for the acceptance of social robots. Robotics and Autonomous Systems, 16: 1476-1486. (2013).

[12] G. Hackbarth, V. Grover, M.Y. Yi. Computer playfulness and anxiety: Positive and negative mediators of the system experience effect on perceived ease of use. Information \& Management, 40: 221232. (2003)

[13] P.A. Hancock, D.R. Billings, K.E. Scheafer, J.Y.C. Chen, E.J. de Visser, R. Parasuraman. A meta-analysis of factors affecting trust in human-robot interaction. Human Factors: The J. of Human Factors and Ergonomics, 53: 517-527. (2011).

[14] M. Heerink, B. Kröse, V. Evers, B. Wielings. Assessing acceptance of assistive social agent technology by older adults: The Almere model. Int. J. of Social Robotics, 2: 361-375. (2010).
[15] D. Hynes, H. Richardson. What use is domestication theory to information system research?. In Y.K. Dwivedi, B. Lal, S.L. Schneberger, \& M. Wade (eds.). Handbook of research contemporary theoretical models in information system (pp. 432494), Information Science Reference, New York, NY, USA. (2009).

[16] E. Karahanna, M. Limayem. E-mail and V-mail usage: Generalizing across technologies. J. of Organizational Computing and Electronic Commerce, 10: 49-66. (2000).

[17] E. Karapanos, J. Zimmerman, J. Forlizzi, J.B. Martens. User experience over time: An initial framework. Proceedings of the Int. Conf. on Human Factors in Computing Systems, Boston, MA, USA. (2009).

[18] A. Kim, J. Han, Y. Jung, K. Lee. The effects of familiarity and robot gesture on user acceptance of information. Proc. of the Int. Conf. on Human-Robot Interaction, Tokyo, Japan. (2013).

[19] R. LaRose, M.S. Eastin. A social cognitive theory of internet uses and gratifications: Toward a new model of media attendance. $J$. of Broadcasting \& Electronic Media, 48: 358-377. (2004).

[20] K. Lee, N. Park, H. Song. Can a robot be perceived as a developing creature?: Effects of a robot's long-term cognitive developments on its social presence and people's social responses toward it. Human Communication Research, 31: 538-563. (2005).

[21] I. Leite, C. Martinho, A. Paiva. Scoial robots for long-term interaction: A survey. Int. J. of Social Robotics, 5: 291-308. (2013)

[22] M. Lohse. Bridging the gap between users' expectations and system evaluations. Proc. of the Int. Symposium on Robot and Human Interactive Communication, Atlanta, GA, USA. (2011).

[23] J.C. McCroskey, J.J. Teven. Goodwill: A re-examination of the construct and its measurement. Communication Monographs, 66: 90 103. (1999).

[24] T. Mirlacher, R. Buchner, F. Förster, A. Weiss, \& M. Tscheligi. Ambient rabbits likeability of embodied ambient displays. Proc. of the European Conference of Ambient Intelligence, Salzburg, Austria. (2001).

[25] J.W. Moon, J.G. Kim. Extending the TAM for a world-wide-web context. Information \& Management, 38: 217-230. (2001).

[26] A.M. von der Pütten, N.C. Krämer, S.C. Eimler. Living with a robot companion. Proceedings of the Int. Conf. on Multimodal Interfaces, Alicante, Spain. (2011).

[27] P.L.P. Rau, Y. Li, D.J. Rau. Effects of communication style and culture on ability to accept recommendations from robots. Computers in Human Behavior, 25: 587-595. (2009).

[28] E.M. Rogers. Diffusion of innovations. The Free Press, New York, NY, USA. (2003)

[29] R. Silverstone, L. Haddon. Design and the domestication of ICT's: Technical change and everyday life. In R. Silverstone and R. Mansell (eds.). Communication by design: The politics of information and communication technologies (pp. 44-74). Oxford Press, Oxford, UK. (1996).

[30] J.Y. Sung, H.I. Christensen, R.E. Grinter. Robots in the wild: Understanding long-term use. Proc. of the Int. Conf. on Human Robot Interaction, La Jolla, CA, USA. (2009)

[31] J.Y. Sung, R.E. Grinter, H.I. Christensen. Domestic robot ecology: An initial framework to unpack long-term acceptance of robots at home. International Journal of Social Robotics, 2: 417-429. (2010).

[32] S. Taylor, P.A. Todd. Understanding information technology usage: A test of competing models. Information System Research, 6: 144176. (1995).

[33] V. Venkatesh. Determinants of ease of use: Integrating control, intrinsic motivation, and emotion into the technology acceptance model. Information Systems Research, 11: 342-365. (2000).

[34] V. Venkatesh, F.D. Davis. A theoretical extension of the technology acceptance model: Four longitudinal field studies. Management Science, 46: 186-204. (2000). 